\title{
The microvascular and morphostructural changes of nails in psoriatic patients with nail disease; a link between ultrasound and videocapil- laroscopy findings in the nailfold
}

\author{
Sibel Bakirci Ureyen', Rabia Oztas Kara², Zeynep Erturk ${ }^{3}$, Mahizer Yaldiz ${ }^{2}$
}

${ }^{1}$ Department of Internal Medicine, Division of Rheumatology, ${ }^{2}$ Department of Dermatology, ${ }^{3}$ Department of Internal Medicine, Sakarya Education and Research Hospital, Sakarya, Turkey

\begin{abstract}
Objective: The objective of this study is to evaluate the link between nail fold vessel resistive index (NVRI) measured by ultrasound (US) and capillary loops diameters measured using nailfold videocapillarascopy (NVC), and to assess the morphological appearance of the nail bed in patients with psoriatic nail disease (PND) as compared with healthy controls (HCs). Material and methods: This study was conducted in patients with PND and HCs. General demographic data were collected and clinical assessments were performed for all subjects. The nail plate thickness (NPT) was measured on gray scale using US. The NVRI was measured using color Doppler (CD) US. The measurements of the apical, arterial, venous limb diameters and morpho-structural changes (tortuous, cross-linked capillaries) were assessed using NVC. Results: Thirty-four patients with PND and $15 \mathrm{HCs}$ were enrolled in this study. The two groups were matched for age and body mass index (BMI). Patients with PND had higher NPT and NVRI in comparison with HCs [(20 (17-23) vs 14 (14-15), p<0.001), (0.55 (0.51-0.61) vs 0.43 $(0.38-0.49), \mathrm{p}<0.001)$, respectively]. A higher proportion of patients with PND had tortuous capillaries than $\mathrm{HCs}(62 \%$ and $20 \%$ respectively, $\mathrm{p}=0.005)$. The mean NVRI was higher in patients with PND who had tortuous capillaries than patients who did not have tortuous capillaries $(0.58(0.7)$ and $0.52(0.09)$, respectively $\mathrm{p}=0.033)$. Conclusion: Microvascular changes can be detected easily using non-invasive methods such as US and NVC. These methods can provide an objective data to better assess PND.
\end{abstract}

Keywords: psoriasis; nail; ultrasound; capillaroscopy; nailfold resistive index

\section{Introduction}

Psoriasis is a chronic, relapsing, immune-mediated inflammatory skin disease, affecting approximately $1 \%$ to $3 \%$ of the population [1]. Nail involvement occurs in approximately $10 \%$ to $55 \%$ of patients with psoriasis but is an often-overlooked clinical manifestation of the dis-

Received 06.10.2017 Accepted 29.12.2017

Med Ultrason

2018, Vol. 20, No 2, 185-191

Corresponding author: Sibel Bakirci Ureyen

Sakarya Education and Research Hospital,

Department of Internal Medicine,

Division of Rheumatology

Şirinevler Mahallesi, Adnan Menderes Cad.

Sağlik Sok No:195

54100 Adapazarı/Sakarya, Türkiye

Phone: +90 4445 400, Fax: 0 (264) 2552105

E-mail: bakircisibel@gmail.com ease [2,3]. Van Laborde et al have shown that there is an $80 \%$ to $90 \%$ lifetime incidence of nail involvement in psoriatic patients [4]. The presence of nail involvement of psoriasis is associated with an increase in patient-reported pain complaints, functional impairment and social burden, resulting in a significant restriction of daily activities and quality of life as well as resistance to treatment [5-9].

The diagnosis of psoriatic nail disease (PND) is based on clinical history and physical examination, and the definitive method of diagnosis is histological examination [10]. PND is most commonly diagnosed by physical examination. Biopsy is painful and may cause lasting cosmetic issues, thus different diagnostic methods have been evaluated to better understand PND.

Nailfold videocapillaroscopy (NVC) is a non-invasive technique to assess the microcirculation and increas- 
ingly is used in the assessment of patients with connective tissue disease. Endothelial cells play an important role in pathogenesis of psoriasis as immune-mediated inflammatory features and papillary dermis have multiple blood vessels, as well as they show microvascular changes such as decreased capillary density and avascular areas. Additionally, NVC can provide some information regarding morphostructural changes of capillaries including tortuosity, elongation, dilatation, and crosslinked [11-15]. Several studies with capillaroscopy have been performed in patients with psoriasis, with either nail or joint involvement [16-19]. One of the advantages of NVC is that individual capillaries can be measured, therefore abnormalities can be determined and quantified $[14,20]$.

Ultrasound (US) has been used to identify synovitis, tenosynovitis, enthesitis, and nail fold abnormalities in patients with psoriasis [21]. US is also a non-invasive imaging method which allows viewing the anatomical and physiological details of the nails and provides information about the morphological appearance of the nail lesions, together with changes of the underlying tissue and blood flow alteration [22]. During the past decade, several studies have evaluated the usefulness of US for diagnosis, identification of disease activity and follow-up response to treatment in patients with psoriasis [23-27].

The relationship among psoriasis and nail disease are not fully understood so far, therefore a better understanding of its mechanism is essential. The objective of this study is to evaluate the link between nail fold vessel resistive index (NVRI) measured using US and capillary loops diameters measured using NVC, as well as to assess the morphological appearance of the nail bed in patients with PND as compared with healthy controls (HCs).

\section{Materials and methods}

\section{Study design}

This cross-sectional, single-center study was conducted in patients with PND admitted to the psoriasis unit of the dermatology outpatient clinic in Sakarya University Education and Research Hospital (SUEAH) between 2016 and 2017. This study was approved by the local Ethics Committee at SUEAH (No:16214662/050.01.04/138) and all individuals gave their written informed consent before enrolment. We included adult patients (age $\geq 18$ years), with skin and nail psoriasis who were systemic treatment-naive. Patients who were on topical corticosteroids for psoriasis plaques on the skin were not excluded. Patients who had used any treatment for nail involvement within last 3 months, including any topical treatment agent and patients with clinical psoriatic arthritis or other inflammatory conditions affecting joints were excluded. In order to avoid potential microvascular changes due to other systemic diseases including; patients with arterial hypertension, diabetes mellitus, and smoking history in the last 5 years were also excluded from study. Healthy volunteers working in the SUEAH and patients' immediate acquaintances without kinship were recruited as HCs. The control group consisted of non-smoking, healthy subjects without any arthritis, diabetes mellitus and arterial hypertension. The two groups were matched for age and body mass index (BMI).

\section{Clinical assessment}

General demographic data were collected including age, gender, BMI, duration of psoriasis, the current medications and past medical history. The severity of skin psoriasis was evaluated by the Psoriasis Area and Severity Index (PASI) (range 0-72), psoriasis nail involvement was assessed by the Nail Psoriasis Severity Index (NAPSI), quality of life was assessed by the Dermatology Quality of Life Index (DQOL) (mild $<10$, moderate $=10-20$, severe $>20$ ) [28]. These assessments were performed by a clinician (ZE) who was blinded to NVC and US findings.

\section{Ultrasonography assessment}

US was performed by an experienced sonographer (ROK) blinded to clinical assessments and NVC findings. The US scanner used was a LOGIQ P9 (General Electric Company, United Kingdom), equipped with a 7-13 MHz linear transducer. The imaging parameters for Doppler US were set to increase the detection of lowvelocity, low-volume flows within the nail bed (Color Doppler (CD): PRF 500-1000 Hz, wall filter $25-50 \mathrm{~Hz}$ ). Color gain was maximized for optimal sensitivity while avoiding excessive color noise. Particular attention was paid not to apply pressure on the tissue to avoid blanching of CD signal. Each US examination took 20 minutes. All images were stored and anonymously numbered, before scoring.

The nails were assessed at $24^{\circ} \mathrm{C}$ room temperature after a 20 minute resting period. The US scan was performed with the patient seated and the hands in neutral position. Morphostructural evaluation of the nail (deposits in dorsal nail plate, irregularities and thickness of nail plates and nail bed thickness) was assessed by gray scale (GS). GS findings were scored by proper scoring system [29]. The measurement of nail plate thickness (NPT) was performed on the right $2^{\text {nd }}$ finger. NPT was measured as the maximum distance between the dorsal and ventral nail plates and GS changes in psoriatic nails were categorized into four types: type1-focal hyperechoic involvement of the ventral plate with or without involvement of 


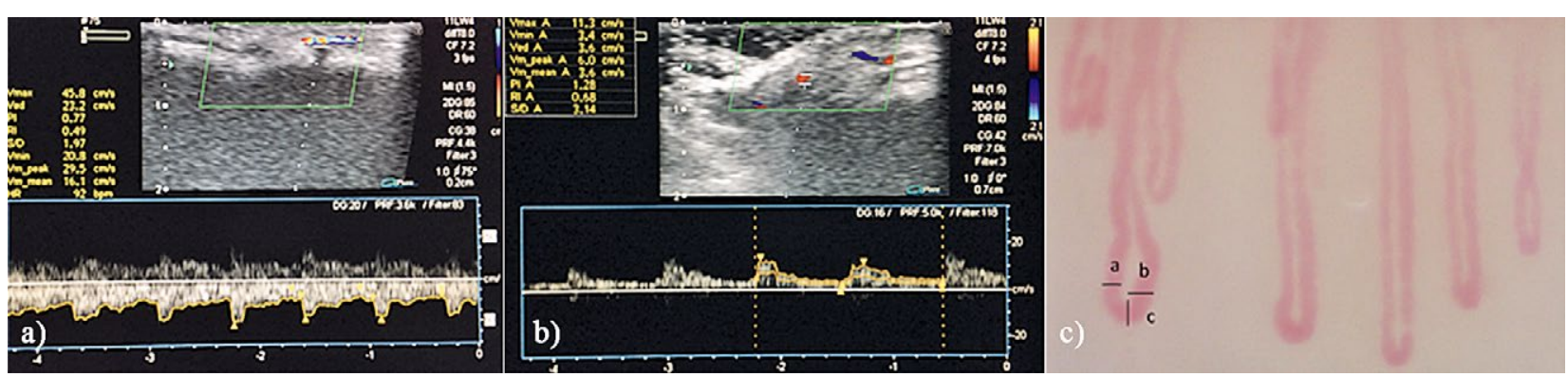

Fig 1. Color Doppler ultrasound, longitudinal view- normal NVRI in healthy controls (a) and increased NVRI in patients with psoriatic nail disease (b); c) The measurement of the diameter of arterial - a, venous - b, and apical - c in nail fold videocapillarascopy.

the dorsal plate; type 2-loosening of the borders of the ventral plate; type3-appearance of wavy plates; type4loss of definition on both plates [29].

NVRI was measured on the $4^{\text {th }}$ finger of the nondominant hand by CD US (fig 1a,b).The CD signal with Spectral Doppler analysis was used to obtain a graphical representation of blood flow over time. The maximum speeds of flow in systole (Qs) and in diastole (Qd) were recorded. NVRI was reported as the following ratio: (QsQd)/Qs. Three measures were obtained for each patient and the arithmetic mean was considered as the definitive NVRI. The NVRI ratio ranged from 0 (no resistance to blood flow) to 1 (maximum resistance to blood flow) [30].

\section{Nailfold videocapillaroscopy assessment}

$\mathrm{NVC}$ was performed by an experienced rheumatologist (SBU) blinded to the results of clinical examination and US findings. The machine used was a Dino-lite capillaroscope device (Dinocapture 2.0 windows software) (magnification $\times 500$ ). However, all images were converted to the same calibration as seen in a capillaryscope device x 200 magnification lenses. NVC device consists of the combination of an optical microscope with a digital video camera that can be attached to any computer. A drop of immersion oil was placed on the cuticle of the fingers to better visualize the capillaries and reduce refractive defects.

The patient undergoing the exam was initially seated in an acclimatized room for 15-20 minutes, with a temperature set around $22-23^{\circ}$ for the prevention of temperature related changes in the vessel wall [31]. Apical, arterial, venous limb diameters were measured on the $4^{\text {th }}$ finger of the non-dominant hand, as it is less subject to trauma. NVC was applied to 8 fingers (excluding the thumbs). Some capillaroscopic parameters (tortuous, cross-linked) were recorded $(1 \times 1 \mathrm{~mm}$ in size) from the middle of the nailfold in each finger. These measurements were taken from the thickest part of the capillary loops (fig 1c). Cross-linked capillaries were describes as similar to braches that intersect like a number eight and tortuous like capillaries with branches in an undulating, sinuous or twisted arrangement [14,32-35]. The capillaroscopic changes (tortuous, cross-linked capillary) were scored as "absent" or "present" as described previously [33]. If more than $50 \%$ of the capillaries in a nailfold were affected, the given abnormality was scored as "present". After all images were stored, they were anonymously numbered, before scoring.

\section{Statistical analysis}

Continuous data were described as mean (standard deviations (SD)) or median (first and third quartile) according to the distribution and categorical variables were expressed as frequencies and percentages. Comparisons between the 2 categories were made using 2-tailed $t$ tests for normally distributed data or the Mann-Whitney $U$ test for non-normally distributed variables. The MannWhitney U test was used for comparing US and NVC findings. The Chi-square test was used to compare categorical variables. $P$ values less than 0.05 were considered statistically significant. SPSS V-15 was used for analysis (SPSS Inc., Chicago, IL, USA).

\section{Results}

Thirty-four patients with PND (16 female and 18 male) and fifteen HCs (13 female and 2 male) were consecutively recruited for this study. A diagnosis of psoriasis was made by an experienced dermatologist (ROK) on the basis of clinical findings. Demographics and disease characteristics of patients were summarized in Table I.

\section{Nailfold videocapillarascopy findings}

No statistical differences were observed between patients with PND and HCs with respect to arterial limb, venous limb, and apical diameters of capillaries.

Analyzing the patients depending on PASI $(<6$ and $\geq 6$ ) we found no statistical differences between the two groups concerning the arterial limb, venous limb, and apical diameters of capillaries $(p=0.968, p=0.887, p=0.839$, respectively). 
Table I. Demographics of patients with psoriatic nail disease and healthy controls

\begin{tabular}{llll}
\hline & Patients with PND (n=34) & HCs (n=15) & p value \\
\hline Age (years) & $42.17(10.5)$ & $40.7(5.1)$ & 0.769 \\
Male/female & $18 / 16$ & $2 / 13$ & 0.009 \\
BMI $\left(\mathrm{kg} / \mathrm{m}^{2}\right)$ & $27.1(7.1)$ & $26.3(3.2)$ & 0.828 \\
Disease duration (years) & $13.8(7.7)$ & - & - \\
PASI & $4.2(2.7)$ & - & - \\
NAPSI & $24.0(18.5-7.5)^{*}$ & - & - \\
DQOL & $7.5(4.5)$ & - & - \\
\hline
\end{tabular}

The results are expressed as mean (standard deviations) or median (first and third quartile)*. PND - psoriatic nail disease; HCs - healthy controls; $\mathrm{n}$ - number of patients; BMI - body mass index; PASI - psoriasis activity severity index; NAPSI - Nail Psoriasis Severity Index; DQOL - Dermatology Quality of Life Index

Table II. Comparison between patients with NVRI $<0.6$ and NVRI $\geq 0.6$ regarding the arterial, venous limb, and apical diameters of capillaries.

\begin{tabular}{llll}
\hline & NVRI $<\mathbf{0 . 6}$ & NVRI $\geq \mathbf{0 . 6}$ & p value \\
\hline Arterial diameters & $25.3(19.4-32.3)$ & $24.8(22.2-28.5)$ & 0.943 \\
Venous diameters & $31.8(23.7-36.7)$ & $31.1(25.7-39.4)$ & 0.576 \\
Apical diameters & $32.6(24.2-39.8)$ & $31.1(25.7-39.4)$ & 0.576 \\
\hline
\end{tabular}

The results are expressed as median (first and third quartile); NVRI- nailfold vessel resistive index

Table III. US findings and NVC findings of all subjects

\begin{tabular}{llll}
\hline & Patients with PND (n=34) & HCs(n=15) & p value \\
\hline Arterial limb diameter (NVC) $\mu \mathrm{m}^{*}$ & $25.3(21.2-29.7)$ & $21.2(19.1-29.9)$ & 0.448 \\
Venous limb diameter (NVC) $\mu \mathrm{m}^{*}$ & $31.8(24.7-37.2)$ & $27.4(24-35)$ & 0.649 \\
Apical diameters (NVC) $\mu \mathrm{m}^{*}$ & $32.6(26.6-36.6)$ & $36.6(27.8-42.1)$ & 0.329 \\
Tortuous capillaries n (\%) & $22(62)$ & $3(20)$ & 0.005 \\
Cross-linked capillaries n (\%) & $9(25)$ & $6(40)$ & 0.333 \\
NPT mm* & $20(17-23)$ & $14(14-15)$ & $<0.001$ \\
NVRI* & $0.55(0.51-0.61)$ & $0.43(0.38-0.49)$ & $<0.001$ \\
\hline
\end{tabular}

The results are expressed as median (first and third quartile)* or number (percent \%). NVC - nailfold videocapillarascopy; PND - psoriatic nail disease; HCs - healthy controls; $n$ - number of patients; NPT - nail plate thickening; NVRI - nailfold vessel resistive index

Table IV. NAPSI, NAPSI nail bed and NAPSI nail matrix compared to PASI $<6$ and PASI $\geq 6$ in patients with PND

\begin{tabular}{llll}
\hline & PASI $<\mathbf{6}$ & PASI $\geq \mathbf{6}$ & p value \\
\hline NAPSI & $23.5(17.7-30.2)$ & $33(13.7-56.2)$ & 0.238 \\
NAPSI nail bed & $17.0(10.5-22)$ & $22(10.2-39)$ & 0.230 \\
NAPSI nail matrix & $7(3.7-10)$ & $9(2-29.7)$ & 0.618 \\
\hline
\end{tabular}

The results are expressed as median (first and third quartile); NAPSI - Nail Psoriasis Severity Index; PASI - psoriasis activity severity index

There was also no statistical difference between the arterial limb, venous limb, and apical diameters when the patients were divided into 2 groups depending on NVRI $(<0.6$ and $\geq 0.6)$ (Table II).

\section{Ultrasound findings}

Patients with PND had higher NPT and NVRI than HCs [ 20 (17-23) vs 14 (14-15), $\mathrm{p}<0.001),(0.55$ (0.51$0.61)$ vs $0.43(0.38-0.49), \mathrm{p}<0.001$, respectively]. Patients with PND were categorized into two groups by NPT measured by US as NPT $<20 \mathrm{~mm}(\mathrm{n}=16)$ and NPT $\geq 20 \mathrm{~mm}(\mathrm{n}=18)$ and the median NAPSI in two groups were $20.5(12.2-33)$ and $28.5(22-35)$, respectively $(\mathrm{p}=$ 0.05 ). There were 6 patients in type $1 \mathrm{GS}$ changes of psoriatic nails, 6 patients in type 2, 20 patients in type 3 , and 2 patients in type 4 . The US findings and NVC findings were summarized in Table III.

\section{The comparisons of clinical assessments}

No statistical difference was observed when NAPSI, NAPSI nail bed, and NAPSI nail matrix were compared in patients with $\mathrm{PASI}<6$ and $\mathrm{PASI} \geq 6$ (Table IV). 
Patients with PND, DLQI $0-9$, and $\geq 10$ were compared with respect to NAPSI scores and no significant difference was observed $(p=0.618)$.

\section{Discussions}

To the best of our knowledge, this is the first study to evaluate the link between microvascular nailfold changes detected with NVC and NVRI findings in patients with PND. Patients with PND had a higher resistance to blood flow, higher thickening of the nail plate and higher tortuous capillary loops in comparison with HCs. Furthermore, NVRI was higher in PND patients with tortuous capillaries. There has been an increasing interest in the last decade to diagnose earlier the nail involvement of psoriasis, after the link between psoriatic arthritis and nail disease were discovered. US provides high quality information regarding both structural and blood flow changes in the nail unit. The ventral nail plate deposits were seen in $17.72 \%$ of involved nails and $4.62 \%$ of the nails without clinical signs of involvement $(\mathrm{p}<0.05)$ [36]. The dorsal and ventral nail plates appear as bilaminar, parallel, hyperechoic lines with a hypoechoic space in between in healthy subjects. The US features of nail psoriasis are represented as the thickening of the nail, poorly defined ventral plate with focal hyperechoic areas, and thickening of both ventral and dorsal plates [37].

Espinoza et al reported endothelial cell swelling and thickening of the vessel wall in patients with psoriasis due to immune complex deposition and activation [38]. El-Ahmed et al illustrated that endothelial cell dysfunction that triggers inflammatory response and thickening of the vessel wall is associated with increased resistance in the bloodstream and decreased blood flow in patients with psoriasis [30]. In our study, patients with psoriasis had a higher NPT in comparison with HCs $(\mathrm{p}<0.001)$. Gisondi et al and Marina et al found similar results in their studies [36,39]. Patients with PND had a significantly higher NVRI than HCs in the present study $(p<0.001)$. Similar NVRI results were reported by El-Ahmed et al and Marina et al $[30,36]$. These results can be explained by the decreased blood supply in the nail bed in psoriatic patients due to endothelial dysfunction and wall vessel thickening [36]. Peripheral vascular resistance is related to vessel length and blood viscosity and the major determinant of resistance is the vessel diameter $[30,40]$. Vascular changes are implicated in the pathogenesis of PND and it is suggested that increased NVRI can play a role in nail involvement.

Errichetti et al have highlighted the usefulness of nail fold and elbow dermoscopy to differentiate early PsA sine psoriasis from early RA in terms of typical vascular find- ings such as dotted vessels [41]. Cross-linked and tortuous capillaries representing microvascular changes have been observed in a number of studies in patients with psoriasis $[11,16,18,40]$. Several authors also observed that psoriatic patients have short capillaries by capillaroscopic examination [16-18]. In our study, patients with PND had a higher frequency of tortuous capillaries in comparison with HCs. We additionally, observed a mild deterioration of quality of life in patients with PND in this study.

This study had some limitations such as small sample size and the use of a medium frequency probe for US. Further studies with a large sample size are required to support these results. Another limitation of this study was that the sonographers could not be blinded due to the need to visualize the clinical condition of the nail while performing the scan; therefore, all images stored and were anonymously numbered, before scoring. Before the US scan started, all subjects were asked to avoid any conversation with the sonographer regarding their diagnosis.

The presence of nail involvement in psoriatic patients was related with elevated vessel resistance and the presence of tortuous capillaries. These findings highlighted the involvement of the nail fold vessel in PND patients

In conclusion, the microvascular changes in patients with PND can be easily detected using non-invasive methods such as US and NVC. These methods can provide objective means to better understand microvascular changes of PND. Based on these findings, further longitudinal studies are necessary to understand the significance and implications of microvascular changes in the psoriatic nail.

\section{Conflict of interest: none}

Acknowledgments: We would like to thank Dr Ali Akdogan and Koray Tascilar for their valuable criticism and their assistance in improving our manuscript.

\section{Reference}

1. Nestle FO. Psoriasis. Curr Dir Autoimmun 2008;10:65-75.

2. Salaffi F, De Angelis R, Grassi W; MArche Pain Prevalence; INvestigation Group (MAPPING) Study. Prevalence of musculoskeletal conditions in an Italian population sample: results of a regional community-based study, I: the MAPPING study. Clin Exp Rheumatol 2005;23:819-828.

3. Salomon J, Szepietowski JC, Proniewicz A. Psoriatic nails: a prospective clinical study. J Cutan Med Surg 2003;7:317321.

4. Van Laborde S, Scher RK. Developments in the treatment of nail psoriasis, melanonychia striata, and onychomycosis. A review of the literature. Dermatol Clin 2000;18:37-46. 
5. Gupta AK, Cooper EA. Psoriatic nail disease: quality of life and treatment. J Cutan Med Surg 2009;13:S102-S106.

6. Reich A, Szepietowski JC. Health-related quality of life in patients with nail disorders. Am J Clin Dermatol 2011;12:313-320.

7. Baran R. The burden of nail psoriasis: an introduction. Dermatology 2010;221:1-5.

8. Schons KR, Beber AA, Beck Mde O, Monticielo OA. Nail involvement in adult patients with plaque-type psoriasis: prevalence and clinical features. An Bras Dermatol 2015;90:314-319.

9. Schons KR, Knob CF, Murussi N, Beber AA, Neumaier W, Monticielo OA. Nail psoriasis: a review of the literature. An Bras Dermatol 2014;89:312-317.

10. Selkin B, Rajadhyaksha M, Gonzalez S, Langley RG. In vivo confocal microscopy in dermatology. Dermatol Clin 2001;19:369-377.

11. Ribeiro CF, Siqueira EB, Holler AP, Fabrício L, Skare TL. Periungual capillaroscopy in psoriasis. An Bras Dermatol 2012;87:550-553.

12. Gallucci F, Russo R, Buono R, Acampora R, Madrid E, Uomo G. Indications and results of videocapillaroscopy in clinical practice. Adv Med Sci 2008;53:149-157.

13. Kayser C, Andrade LEC. Capilaroscopia periungueal: importância para a investigação do fenômeno de Raynaud e doenças do espectro da esclerose sistêmica. Rev Bras Reum 2004;44:16-52.

14. Grassi W, De Angelis R. Capillaroscopy: questions and answers. Clin Rheumatol 2007;26:2009.

15. Braverman IM, Yen A. Ultrastructure of the capillary loops in the dermal papillae of psoriasis. J Invest Dermatol 1977;68:53-60.

16. Zaric D, Clemmensen OJ, Worm AM, Stahl D. Capillary microscopy of the nail fold in patients with psoriasis and psoriatic arthritis. Dermatologica 1982;164:10-14.

17. Ohtsuka T, Yamakage A, Miyachi Y. Statistical definition of nailfold capillary pattern in patients with psoriasis. Int J Dermatol 1994;33:779-782.

18. Zaric D, Worm AM, Stahl D, Clemmensen OJ. Capillary microscopy of the nailfold in psoriatic and rheumatoid arthritis. Scand J Rheumatol 1981;10:249-252.

19. Grassi W, Core P, Carlino G, Cervini C. Nailfold capillary permeability in psoriatic arthritis. Scand J Rheumatol 1992;21:226-230.

20. Hern S, Mortimer PS. In vivo quantification of microvessels in clinically uninvolved psoriatic skin and in normal skin. Br J Dermatol 2007;156:1224-1229.

21. Zabotti A, Bandinelli F, Batticciotto A, et al; Musculoskeletal Ultrasound Study Group of the Italian Society of Rheumatology. Musculoskeletal ultrasonography for psoriatic arthritis and psoriasis patients: a systematic literature review. Rheumatology 2017;56:1518-1532.

22. Machet L, Ossant F, Bleuzen A, Grégoire JM, Machet MC, Vaillant L. High-resolution ultrasonography: utility in diagnosis, treatment, and monitoring of dermatologic diseases. J Radiol 2006;87:1946-1961.
23. Gupta AK, Turnbull DH, Harasiewicz KA, et al. The use of high-frequency ultrasound as a method of assessing the severity of a plaque of psoriasis. Arch Dermatol 1996;132:658-662.

24. Vaillant L, Berson M, Machet L, Callens A, Pourcelot L, Lorette G. Ultrasound imaging of psoriatic skin: a noninvasive technique to evaluate treatment of psoriasis. Int $J$ Dermatol 1994;33:786-790.

25. El Gammal S, El Gammal C, Kaspar K, et al. Sonography of the skin at $100 \mathrm{MHz}$ enables in vivo visualization of stratum corneum and viable epidermis in palmar skin and psoriatic plaques. J Invest Dermatol1999;113:821-829.

26. El Gammal S, Auer T, Popp C, et al. Psoriasis vulgaris in 50 $\mathrm{MHz}$ B-scan ultrasound: characteristic features of stratum corneum, epidermis and dermis. Acta Derm Venereol Suppl (Stockh) 1994;186:173-176.

27. Olsen LO, Serup J. High-frequency ultrasound scan for noninvasive cross-sectional imaging of psoriasis. Acta DermVenereol 1993;73:185-187.

28. Mease PJ. Measures of psoriatic arthritis: Tender and Swollen Joint Assessment, Psoriasis Area and Severity Index (PASI), Nail Psoriasis Severity Index (NAPSI), Modified Nail Psoriasis Severity Index (mNAPSI), Mander/ Newcastle Enthesitis Index (MEI), Leeds Enthesitis Index (LEI), Spondyloarthritis Research Consortium of Canada (SPARCC), Maastricht Ankylosing Spondylitis Enthesis Score (MASES), Leeds Dactylitis Index (LDI), Patient Global for Psoriatic Arthritis, Dermatology Life Quality Index (DLQI), Psoriatic Arthritis Quality of Life (PsAQOL), Functional Assessment of Chronic Illness Therapy-Fatigue (FACIT-F), Psoriatic Arthritis Response Criteria (PsARC), Psoriatic Arthritis Joint Activity Index (PsAJAI), Disease Activity in Psoriatic Arthritis (DAPSA), and Composite Psoriatic Disease Activity Index (CPDAI). Arthritis Care Res (Hoboken) 2011;63:S64-S85.

29. Gutierrez M, Wortsman X, Filippucci E, De Angelis R, Filosa G, Grassi W. High-frequency sonography in the evaluation of psoriasis: nail and skin involvement. J Ultrasound Med 2009;28:1569-1574.

30. Husein El-Ahmed H, Garrido-Pareja F, Ruiz-Carrascosa JC, Naranjo-Sintes R. Vessel resistance to blood flow in the nailfold in patients with psoriasis: a prospective case-control echo Doppler-based study. Br J Dermatol 2012;166:54-58.

31. Cutolo M, Sulli A, Secchi ME, Olivieri M, Pizzorni C. The contribution of capillaroscopy to the differential diagnosis of connective autoimmune diseases. Best Pract Res Clin Rheumatol 2007;21:1093-1108.

32. Cutolo M, Pizzorni C, Sulli A. Capillaroscopy. Best Pract Res Clin Rheumatol 2005;19:437-452.

33. Grassi W, Del Medico P. Atlas of Capillaroscopy. Milan: Edra Medical Publishing \& New Media. 2004.

34. Cutolo M, Grassi W, Matucci Cerinic M. Raynaud's phenomenon and the role of capillaroscopy. Arthritis Rheum 2003;48:3023-3030.

35. Lambova S, Hermann W, Muller-Ladner U. Capillaroscopic pattern at the toes of systemic sclerosis patients: 
does it 'tell' more than those of fingers? J Clin Rheumatol 2011;17:311-314.

36. Marina ME, Solomon C, Bolboaca SD, Bocsa C, Mihu CM, Tătaru AD. High-frequency sonography in the evaluation of nail psoriasis. Med Ultrason 2016;18:312-317.

37. Wortsman X, Jemec GB. Ultrasound imaging of nails. Dermatol Clin 2006;24:323-328.

38. Espinoza LR, Vasey FB, Espinoza CG, Bocanegra TS, Germain BF. Vascular changes in psoriatic synovium. A light and electron microscopic study. Arthritis Rheum 1982;25:677-684.
39. Gisondi P, Idolazzi L, Girolomoni G. Ultrasonography reveals nail thickening in patients with chronic plaque psoriasis. Arch Dermatol Res 2012;304:727-732.

40. Creamer D, Allen MH, Sousa A, Poston R, Barker JN. Localization of endothelial proliferation and microvascular expansion in active plaque psoriasis. Br J Dermatol 1997; 136:859-865.

41. Errichetti E, Zabotti A, Stinco G, et al. Dermoscopy of nail fold and elbow in the differential diagnosis of early psoriatic arthritis sine psoriasis and early rheumatoid arthritis. J Dermatol 2016;43:1217-1220. 